\title{
Atezolizumab plus Bevacizumab versus Sorafenib in the Chinese Subpopulation with Unresectable Hepatocellular Carcinoma: Phase 3 Randomized, Open-Label IMbrave150 Study
}

\author{
Shukui Qin ${ }^{\mathrm{a}}$ Zhenggang Ren ${ }^{\mathrm{b}}$ Yin-Hsun Feng ${ }^{\mathrm{c}}$ Thomas Yau $^{\mathrm{d}}$ \\ Baocheng Wang ${ }^{\text {e Haitao Zhao }}{ }^{f}$ Yuxian Baig Shanzhi Gu ${ }^{\text {h }}$ Lindong $\mathrm{Li}^{\mathrm{i}}$ \\ Sairy Hernandez ${ }^{j}$ Derek-Zhen $\mathrm{Xu}^{i}$ Sohail Mullak ${ }^{k}$ Yifan Wang ${ }^{i}$ Hui Shao ${ }^{i}$ \\ Ann Lii Cheng'

\begin{abstract}
aPeople's Liberation Army Cancer Center, Jinling Hospital, Nanjing, PR China; biver Cancer Institute and Department of Liver Cancer Surgery, Zhongshan Hospital, Fudan University, Shanghai, PR China; 'Department of Internal Medicine, Chi Mei Medical Center, Tainan, Taiwan; 'Department of Medicine, Haematology, Medical Oncology \& Bone Marrow Transplantation Division, Queen Mary Hospital, Hong Kong, PR China; 'Department of Oncology, General Hospital of Jinan Military Command, Jinan, PR China; fDepartment of Liver Surgery, Peking Union Medical College Hospital, Beijing, PR China; '9Department of Gastrointestinal Oncology, Harbin Medical University Cancer Hospital, Harbin, PR China; hadioactive Interventional Department, Hunan Cancer Hospital, Changsha, PR China; iProduct Development Oncology, Roche (China) Holding Ltd., Shanghai, PR China; jMedical Mississauga, ON, Canada; 'Department of Medical Oncology, National Taiwan University Cancer Center and
\end{abstract} \\ Affairs, Genentech, Inc., South San Francisco, CA, USA; kProduct Development Biometrics, Hoffmann-La Roche Ltd., \\ National Taiwan University Hospital, Taipei, Taiwan
}

\section{Keywords}

Systemic treatment - Chinese patients - Overall survival . Programmed death-ligand 1 inhibitor - Checkpoint inhibitor · Immunotherapy · Liver cancer

\footnotetext{
Abstract

Introduction: Atezolizumab plus bevacizumab significantly improved overall survival (OS) and progression-free survival (PFS) versus sorafenib in patients with unresectable hepatocellular carcinoma (HCC) in IMbrave150. Efficacy and safety data from the Chinese subpopulation are reported. Methods: IMbrave150, a global, randomized, open-label, phase 3 study in patients with systemic treatment-naive unresect-
}

able HCC, included an extension phase that enrolled additional patients from mainland China. Patients were randomized (2:1) to receive intravenous atezolizumab 1,200 mg plus bevacizumab $15 \mathrm{mg} / \mathrm{kg}$ once every 3 weeks or sorafenib $400 \mathrm{mg}$ twice a day until unacceptable toxicity or loss of clinical benefit. Co-primary endpoints were OS and independent review facility-assessed PFS per Response Evaluation Criteria in Solid Tumors version 1.1 in the intention-totreat population. Results: Of 194 Chinese patients enrolled

These data have previously been presented in part at EASL's Liver Cancer Summit, February 6-8, 2020, in Prague, Czech Republic (oral presentation OP02-03).

ClinicalTrials.Gov Identifier: NCT03434379.
(C) 2021 The Author(s)

Published by S. Karger AG, Basel

This article is licensed under the Creative Commons AttributionNonCommercial-NoDerivatives 4.0 International License (CC BYNC-ND) (http://www.karger.com/Services/OpenAccessLicense). Usage and distribution for commercial purposes as well as any distribution of modified material requires written permission.
Correspondence to:

Ann Lii Cheng, alcheng@ntu.edu.tw 
from April 16, 2018, to April 8, 2019 (137 in the global study and 57 in the China extension phase), 133 received atezolizumab plus bevacizumab and 61 received sorafenib. At the data cutoff (August 29, 2019), the stratified hazard ratio for OS was $0.44(95 \% \mathrm{Cl}, 0.25-0.76)$ and for PFS was 0.60 (95\% $\mathrm{Cl}, 0.40-0.90)$. The respective median OS and PFS with atezolizumab plus bevacizumab were not reached (NR; $95 \%$ $\mathrm{Cl}, 13.5$ months to $\mathrm{NR})$ and 5.7 months $(95 \% \mathrm{Cl}, 4.2-8.3)$ versus 11.4 months ( $95 \% \mathrm{Cl}, 6.7$ to $\mathrm{NR})$ and 3.2 months $(95 \% \mathrm{Cl}$, 2.6-4.8) with sorafenib. Grade 3-4 adverse events (AEs) occurred in 78 of 132 (59.1\%) atezolizumab plus bevacizumabtreated and 27 of $58(46.6 \%)$ sorafenib-treated patients. The most common grade 3-4 AE with atezolizumab plus bevacizumab was hypertension, occurring in $15.2 \%$ of patients; however, other high-grade AEs were infrequent. Conclusion: Clinically meaningful improvements in OS and PFS observed with atezolizumab plus bevacizumab versus sorafenib suggest that atezolizumab plus bevacizumab may become a practice-changing treatment for Chinese patients with unresectable HCC.

(C) 2021 The Author(s) Published by S. Karger AG, Basel

\section{Introduction}

China accounts for more than half of the global incidence of liver cancer cases, of which $70-85 \%$ are hepatocellular carcinoma (HCC) [1]. Hepatitis B virus (HBV) infection is the primary etiological risk factor for HCC in East Asia [2] and is responsible for $64 \%$ of liver cancer deaths in China [3]. By contrast, hepatitis $\mathrm{C}$ virus (HCV) infection is the primary risk factor for HCC in the USA, Europe, and Japan $[4,5]$. Other risk factors for HCC include aflatoxin exposure, alcohol use and smoking (the most common etiologies in China after HBV and HCV [3]), and obesity and nonalcoholic steatohepatitis (more common in the USA and Europe) [4, 5]. Most patients with HCC have concomitant chronic liver disease [6] and present with unresectable disease [7] due to the late emergence of symptoms. These patients have a poor prognosis and short overall survival (OS) [1]. In addition, patients with HBV-related HCC have a significantly worse prognosis than patients with HCV-related HCC and patients with non-HBV and non-HCV HCC $[8,9]$.

Until recently, the multikinase inhibitors sorafenib or lenvatinib were the only recommended first-line systemic treatments after locoregional treatment failure or for patients with advanced HCC $[10,11]$. FOLFOX4 (5-fluorouracil, leucovorin, and oxaliplatin) is also approved for advanced HCC in China, but monitoring of hepatotoxic-

Atezolizumab plus Bevacizumab in Chinese Patients with HCC ity and nephrotoxicity is required [12]. These treatments are associated with considerable toxicities and impaired quality of life (QOL) [13, 14].

Inhibition of programmed death ligand 1 (PD-L1)/ programmed death-1 (PD-1) reverses T-cell suppression, and reversal of immune exhaustion with immune checkpoint inhibitors has been effective in patients with advanced HCC [15-19]. However, phase 3 studies of antiPD-1 immune checkpoint inhibitor monotherapy in first- and second-line settings failed to meet their OS primary endpoints $[20,21]$, suggesting that a combination strategy is necessary to improve clinical efficacy. Antivascular endothelial growth factor (VEGF) therapies reduce VEGF-mediated immunosuppression within the tumor and its microenvironment [22-24] and may enhance anti-PD-L1/PD-1 efficacy by reversing VEGF-mediated immunosuppression and promoting T-cell infiltration in tumors $[25,26]$. A phase $1 \mathrm{~b}$ study of atezolizumab (anti-PD-L1) plus bevacizumab (anti-VEGF) in patients with systemic treatment-naive unresectable HCC demonstrated acceptable tolerability and promising antitumor activity, with a median progression-free survival (PFS) of 7.3 months and a confirmed objective response rate (ORR) of $36 \%$ [19].

The IMbrave150 study of atezolizumab plus bevacizumab was the first randomized phase 3 study since the approval of sorafenib in 2008 to show a statistically significant improvement in OS versus sorafenib in patients with systemic treatment-naive unresectable HCC [27]. Comparing atezolizumab plus bevacizumab with sorafenib, the hazard ratio (HR) for OS was 0.58 (95\% CI, $0.42-0.79 ; p<0.001)$ and the HR for PFS was $0.59(95 \%$ CI, $0.47-0.76 ; p<0.001)$ in the global study population [27]. The median PFS was 6.8 and 4.3 months in the atezolizumab plus bevacizumab and sorafenib arms, respectively [27]. Atezolizumab plus bevacizumab has been approved by the US FDA as treatment for unresectable or metastatic HCC on the basis of these results and is also now recommended as first-line treatment for patients with HCC who have not received prior systemic therapy in the National Comprehensive Cancer Network (NCCN) guidelines [10].

Although HCC is a global disease, geographic heterogeneity is seen in terms of disease etiology, treatment practice, and efficacy outcomes, with shorter median OS observed with sorafenib $[28,29]$ and other multikinase inhibitors $[30,31]$ in Asian versus global populations. This analysis was conducted to determine the efficacy and safety of atezolizumab plus bevacizumab versus sorafenib in the Chinese patients in the global IMbrave150 study, 
as well as in those who enrolled during an additional China extension enrollment phase, who all had unresectable HCC and had not received prior systemic therapy.

\section{Methods}

\section{Study Design}

IMbrave150 (NCT03434379) is a randomized, global, multicenter, open-label, phase 3 study to compare the efficacy and safety of atezolizumab plus bevacizumab versus sorafenib in patients with unresectable HCC who had not received prior systemic treatment [27]. In addition to patients enrolled in the global study population at 111 sites in 17 countries and regions, IMbrave150 included a China extension enrollment phase, into which additional patients were enrolled from mainland China only. The Chinese subpopulation described here thus included all Chinese patients enrolled in mainland China, Hong Kong, and Taiwan at 28 sites during both the global and China extension enrollment phases. Randomization to the atezolizumab plus bevacizumab or sorafenib arms in a 2:1 ratio was performed via an interactive voice-response or Web-response system using permuted blocks stratified by geographic region (Asia [excluding Japan] vs. rest of world [in the global population only]), macrovascular invasion (MVI) and/or extrahepatic spread (EHS; presence vs. absence), baseline $\alpha$-fetoprotein (AFP; $<400$ vs. $\geq 400 \mathrm{ng} / \mathrm{mL}$ ), and Eastern Cooperative Oncology Group (ECOG) performance status (0 vs. 1).

Full eligibility criteria have been previously described [27] and are provided in the protocol (see online suppl. data; for all online suppl. material, see www.karger.com/doi/10.1159/000513486). Briefly, eligible patients were aged $\geq 18$ years, had locally advanced metastatic and/or unresectable HCC, and had not received prior systemic therapy for HCC. They had to have measurable disease per Response Evaluation Criteria In Solid Tumors version 1.1 (RECIST 1.1), which was not amenable to curative or locoregional therapies or had progressed, an ECOG performance status score of 0 or 1, Child-Pugh class A liver function, and esophagogastroduodenoscopy within 6 months prior to enrollment [27]. For the extended China enrollment phase, patients had to have Chinese ancestry and residence in mainland China, Hong Kong, or Taiwan.

Key exclusion criteria included a history of autoimmune disease, fibrolamellar or sarcomatoid HCC or mixed cholangiocarcinoma and HCC, coinfection with HBV and HCV, and untreated or incompletely treated esophageal and/or gastric varices with bleeding or a high risk for bleeding. The study (NCT03434379) was conducted in accordance with local laws. The protocol and amendments were approved by the relevant Institutional Review Boards or Ethics Committee at all centers. All patients provided written informed consent.

\section{Procedures}

Patients received intravenous atezolizumab $1,200 \mathrm{mg}$ plus bevacizumab $15 \mathrm{mg} / \mathrm{kg}$ every 3 weeks or oral sorafenib $400 \mathrm{mg}$ twice daily until they experienced unacceptable toxicity or loss of clinical benefit in the opinion of the investigator. They could continue treatment beyond disease progression if the investigator observed evidence of clinical benefit and no signs indicating unequivocal disease progression. Patients who transiently or permanently discontinued either atezolizumab or bevacizumab due to an adverse event (AE) could remain on single-agent therapy as long as clinical benefit was observed. Dose reductions of atezolizumab or bevacizumab were not permitted, but sorafenib dosage could be reduced to manage AEs.

Tumor assessment by computed tomography or magnetic resonance imaging was conducted at baseline, every 6 weeks for 54 weeks, and every 9 weeks thereafter. Multiphase liver imaging with high-quality imaging of the arterial and portal venous phases was mandatory. Patient-reported outcomes were evaluated using the European Organisation for Research and Treatment of Cancer quality-of-life questionnaire for cancer (EORTC QLQ-C30) at baseline, every 3 weeks until treatment discontinuation or disease progression (whichever came first), and then every 3 months for 1 year.

Safety was continuously evaluated by recording vital signs and clinical laboratory test results and assessing the incidence and severity of AEs according to the National Cancer Institute Common Terminology Criteria for Adverse Events, version 4.0. All AEs were reported until 30 days (90 days for AEs of special interest [AESIs] and serious AEs [SAEs]) after the last dose of study treatment or until the initiation of new systemic anticancer therapy, whichever occurred first.

\section{Outcomes}

The co-primary endpoints were OS (time from randomization to death from any cause) and PFS (time from randomization to disease progression per independent review facility [IRF]-assessed RECIST 1.1 or death from any cause, whichever occurred first). Secondary endpoints included ORR (defined as the percentage of patients who had a confirmed complete or partial response at 2 consecutive tumor assessments $\geq 28$ days apart) per IRF-assessed RECIST 1.1 and HCC-specific modified RECIST (HCC mRECIST) criteria, and time to deterioration (TTD; time from randomization to first decrease from baseline of $\geq 10$ points maintained for 2 consecutive assessments or 1 assessment followed by death from any cause within 3 weeks) in QOL, physical functioning, and role functioning using the EORTC QLQ-C30.

\section{Statistical Analysis}

In the global IMbrave150 study, a sample size of approximately 480 patients (targeting 312 deaths) was planned to provide $80 \%$ power to detect an $\mathrm{HR}$ of 0.71 favoring atezolizumab plus bevacizumab over sorafenib, using a log-rank test at a 2 -sided 0.048 significance level for OS [27]. Within this sample size, a total enrollment of approximately 135 patients from mainland China was planned in line with the requirements of the China Health Authority for the approval of new treatments. If fewer than 135 patients were enrolled from mainland China, additional patients from China could be randomized to the 2 treatment arms (also in a 2:1 ratio) during an extended China enrollment phase. The Chinese subpopulation analyzed here includes all patients enrolled in mainland China (during both the global enrollment phase and the extended China enrollment phase) as well as patients enrolled in Taiwan and Hong Kong during the global enrollment phase.

The analyses described here were conducted at the time of the PFS primary analysis in the global study population. For the coprimary endpoints of OS and IRF-assessed PFS per RECIST 1.1 in the Chinese subpopulation, no formal hypothesis testing was performed because the aim of this analysis was to demonstrate consistency with the global results, not statistical significance for efficacy differences between treatments. 


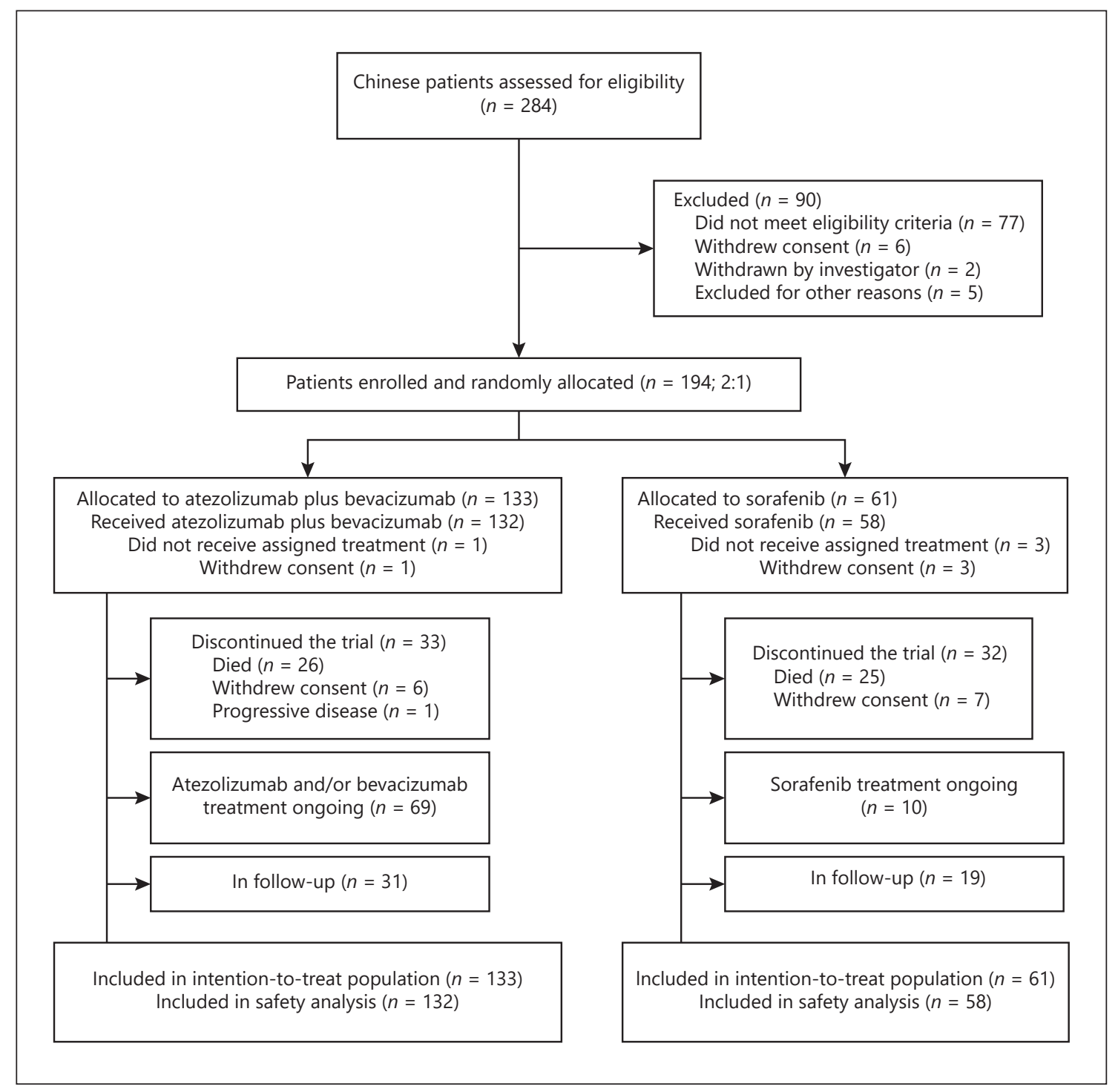

Fig. 1. Trial profile.

The same statistical analysis methods were used for the Chinese subpopulation as for the global study population [27]. The KaplanMeier analysis methodology was applied to OS, PFS, duration of response, and TTD for patient-reported outcomes; HRs and 95\% CIs were estimated using a Cox proportional hazards model stratified by MVI and/or EHS (presence vs. absence) and baseline AFP ( $<400$ vs. $\geq 400 \mathrm{ng} / \mathrm{mL}$ ) (online suppl. data).

All Chinese patients who were randomized to study treatment were evaluable for efficacy. Safety-evaluable patients included those who received $\geq 1$ dose of study treatment. Patient flow through the trial, baseline characteristics, and AEs were summarized by descriptive statistics. SAS (version 9.4; SAS Institute, Cary, NC, USA) was used for all statistical analyses.

Atezolizumab plus Bevacizumab in Chinese Patients with HCC

\section{Results}

A total of 194 Chinese patients were enrolled between April 16, 2018, and April 8, 2019. The Chinese subpopulation included 135 patients from mainland China (of which 78 were enrolled during the global enrollment phase and 57 during the China enrollment phase) and 59 patients enrolled in Taiwan and Hong Kong during the global enrollment phase. Of these, 133 patients were randomized to the atezolizumab plus bevacizumab arm and 61 patients to the sorafenib arm; all were included in the intention-to-treat (ITT) Chinese subpopulation evaluated for efficacy (Fig. 1). At least 1 dose of study treatment 
Table 1. Baseline characteristics of Chinese patients

\begin{tabular}{|c|c|c|}
\hline Characteristics & $\begin{array}{l}\text { Atezolizumab } \\
\text { plus bevacizumab }(n=133)\end{array}$ & $\begin{array}{l}\text { Sorafenib } \\
(n=61)\end{array}$ \\
\hline Median age (range), years & $57(29-82)$ & $60(31-82)$ \\
\hline Age $\geq 65$ years, $n(\%)$ & $32(24.1)$ & $17(27.9)$ \\
\hline Male, $n(\%)$ & $116(87.2)$ & $49(80.3)$ \\
\hline \multicolumn{3}{|l|}{ ECOG performance status, $n(\%)$} \\
\hline 0 & $78(58.6)$ & $31(50.8)$ \\
\hline 1 & $55(41.4)$ & $30(49.2)$ \\
\hline \multicolumn{3}{|l|}{ Child-Pugh score, $n(\%)$} \\
\hline A5 & $107(80.5)$ & $45(73.8)$ \\
\hline A6 & $25(18.8)$ & $16(26.2)$ \\
\hline B7 & 0 & 0 \\
\hline $\mathrm{B} 8$ & $1(0.8)$ & 0 \\
\hline \multicolumn{3}{|l|}{ Barcelona Clinic Liver Cancer stage, $n(\%)$} \\
\hline A & $3(2.3)$ & $1(1.6)$ \\
\hline $\mathrm{B}$ & $15(11.3)$ & $3(4.9)$ \\
\hline $\mathrm{C}$ & $115(86.5)$ & $57(93.4)$ \\
\hline AFP at baseline $\geq 400 \mathrm{ng} / \mathrm{mL}, n(\%)$ & $59(44.4)$ & $30(49.2)$ \\
\hline MVI present, $n(\%)$ & $49(36.8)$ & $28(45.9)$ \\
\hline EHS present, $n(\%)$ & $91(68.4)$ & $40(65.6)$ \\
\hline MVI and/or EHS present, $n(\%)$ & $111(83.5)$ & $53(86.9)$ \\
\hline \multicolumn{3}{|l|}{ Etiology of HCC, $n(\%)$} \\
\hline Hepatitis B & $117(88.0)$ & $47(77.0)$ \\
\hline Hepatitis C & $10(7.5)$ & $7(11.5)$ \\
\hline Nonviral $^{\mathrm{a}}$ & $6(4.5)$ & $7(11.5)$ \\
\hline \multicolumn{3}{|l|}{ Alcohol use, $n(\%)$} \\
\hline Current & $3(2.3)$ & $3(4.9)$ \\
\hline Never & $82(61.7)$ & $37(60.7)$ \\
\hline Previous & $48(36.1)$ & $21(34.4)$ \\
\hline Prior locoregional treatment, $n(\%)^{\mathrm{b}}$ & $87(65.4)$ & $35(57.4)$ \\
\hline Percutaneous ethanol injection & $6(4.5)$ & $1(1.6)$ \\
\hline Radiofrequency ablation & $24(18.0)$ & $15(24.6)$ \\
\hline Transarterial embolization & $8(6.0)$ & $4(6.6)$ \\
\hline Transarterial chemoembolization & $71(53.4)$ & $27(44.3)$ \\
\hline Drug-eluting beads - transarterial chemoembolization & $1(0.8)$ & 0 \\
\hline Transcatheter arterial infusion & $5(3.8)$ & 0 \\
\hline Transarterial radioembolization & $1(0.8)$ & $1(1.6)$ \\
\hline Other & $7(5.3)$ & $4(6.6)$ \\
\hline
\end{tabular}

AFP, a-fetoprotein; MVI, macrovascular invasion; EHS, extrahepatic spread; ECOG, Eastern Cooperative

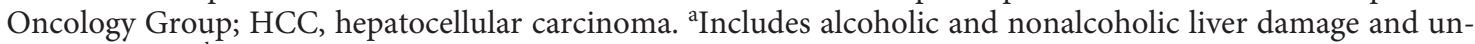

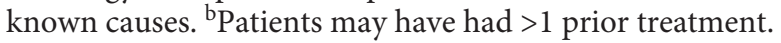

was taken by 132 patients receiving atezolizumab plus bevacizumab and 58 receiving sorafenib, and these patients were included in the safety population.

At the data cutoff on August 29, 2019, the median duration of follow-up was 6.8 months. In the atezolizumab plus bevacizumab arm, 69 patients (51.9\%) were still receiving treatment and $31(23.3 \%)$ were in follow-up (Fig. 1). In the sorafenib arm, 10 (16.4\%) and 19 patients $(31.1 \%)$, respectively, were receiving treatment and in follow-up. The most common reason for study discontinu- ation was death: 26 patients (19.5\%) died in the atezolizumab plus bevacizumab arm and $25(41.0 \%)$ in the sorafenib arm. The baseline characteristics of the Chinese subpopulation are shown in Table 1 and were generally well balanced between the treatment arms. They were generally similar to those in the overall ITT population (online suppl. Table 1), with the following exceptions: the proportion of patients with HBV etiology of HCC was higher in the Chinese subpopulation than in the global population ( 84.5 vs. $47.9 \%$ ), whereas the proportion of 


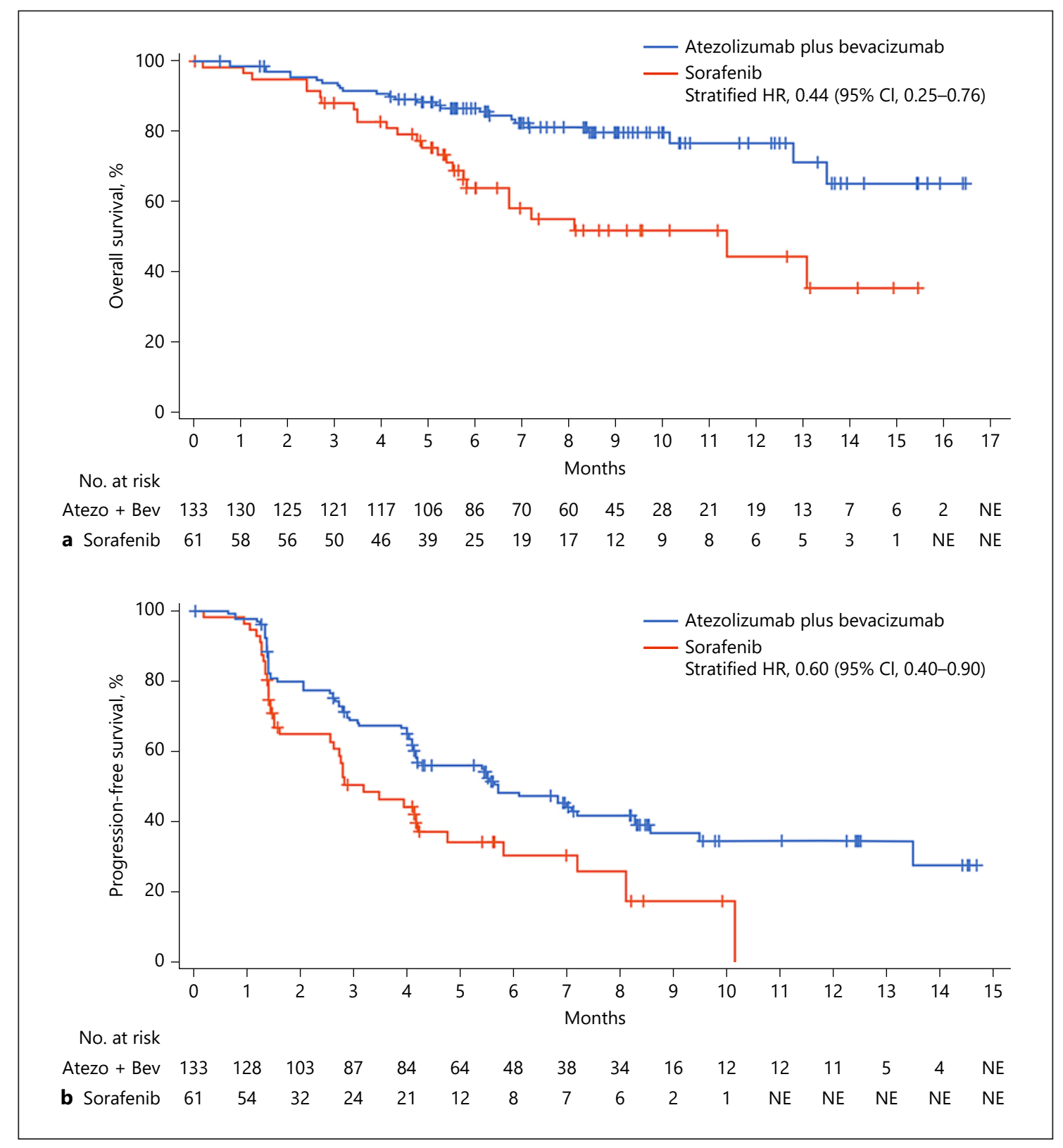

Fig. 2. OS and PFS. Kaplan-Meier analysis of OS (a) and PFS (b) per independent review facility-assessed Response Evaluation Criteria in Solid Tumors 1.1 in the intention-to-treat Chinese subpopulation. OS, overall survival; PFS, progression-free survival; $\mathrm{HR}$, hazard ratio; NE, not estimable.

patients with nonviral etiology (6.7 vs. $30.5 \%)$ and a history of previous and current alcohol use (38.7 vs. $63.5 \%)$ was lower than in the global population. Also, a greater proportion of Chinese than global patients had received prior locoregional treatment (62.9 vs. $49.1 \%$ ), had MVI and/or EHS (84.5 vs. $75.4 \%$ ), AFP $\geq 400 \mathrm{ng} / \mathrm{mL}$ ( 45.9 vs. 37.3\%), and Barcelona Clinic Liver Cancer Stage C (88.7 vs. $81.6 \%)$.

Atezolizumab plus Bevacizumab in Chinese Patients with HCC
The stratified HR for OS was 0.44 (95\% CI, 0.25-0.76) (Fig. 2a). The median OS was not reached in the atezolizumab plus bevacizumab arm (95\% CI, 13.5 months to not reached [NR]) and 11.4 months (95\% CI, 6.7 months to $\mathrm{NR}$ ) in the sorafenib arm. The proportions of patients alive at 6 months were 86.6 and $64.1 \%$ in the respective treatment arms. The proportions of patients alive at 12 months were 76.7 and $44.6 \%$ in the respective treatment arms.

Liver Cancer 2021;10:296-308 
Table 2. Confirmed objective responses to treatment in the intention-to-treat Chinese subpopulation according to different tumor assessment criteria

\begin{tabular}{|c|c|c|c|c|}
\hline \multirow[t]{2}{*}{ Variable } & \multicolumn{2}{|l|}{ IRF RECIST $1.1^{\mathrm{a}}$} & \multicolumn{2}{|c|}{ IRF HCC mRECIST ${ }^{b}$} \\
\hline & $\begin{array}{l}\text { atezolizumab plus } \\
\text { bevacizumab } \\
(n=130)\end{array}$ & $\begin{array}{l}\text { sorafenib } \\
(n=60)\end{array}$ & $\begin{array}{l}\text { atezolizumab plus } \\
\text { bevacizumab } \\
(n=128)\end{array}$ & $\begin{array}{l}\text { sorafenib } \\
(n=59)\end{array}$ \\
\hline Confirmed objective response, $n(\%)$ & $32(24.6)$ & $4(6.7)$ & $38(29.7)$ & $5(8.5)$ \\
\hline $95 \% \mathrm{CI}$ & $17.5-32.9$ & $1.9-16.2$ & $21.9-38.4$ & $2.8-18.7$ \\
\hline Complete response, $n(\%)$ & $5(3.8)$ & 0 & $16(12.5)$ & 0 \\
\hline Partial response, $n(\%)$ & $27(20.8)$ & $4(6.7)$ & $22(17.2)$ & $5(8.5)$ \\
\hline Stable disease, $n(\%)^{\mathrm{c}}$ & $59(45.4)$ & $25(41.7)$ & $52(40.6)$ & $24(40.7)$ \\
\hline Progressive disease, $n(\%)$ & $29(22.3)$ & $19(31.7)$ & $28(21.9)$ & $18(30.5)$ \\
\hline Not evaluable, $n(\%)$ & $4(3.1)$ & $4(6.7)$ & $4(3.1)$ & $4(6.8)$ \\
\hline Missing, $n(\%)$ & $6(4.6)$ & $8(13.3)$ & $6(4.7)$ & $8(13.6)$ \\
\hline Disease control rate, $n(\%)^{\mathrm{d}}$ & $91(70.0)$ & $29(48.3)$ & $90(70.3)$ & $29(49.2)$ \\
\hline Ongoing response at data cutoff, $n(\%)$ & $29(90.6)$ & $3(75.0)$ & $33(86.8)$ & $3(60.0)$ \\
\hline
\end{tabular}

HCC mRECIST, hepatocellular carcinoma-specific modified Response Evaluation Criteria in Solid Tumors; IRF, independent review facility. ${ }^{a}$ Based on patients who presented at baseline with measurable disease per IRF RECIST 1.1. ${ }^{b}$ Based on patients who presented at baseline with measurable disease per IRF HCC mRECIST. ${ }^{\mathrm{C} e}$ quires a designation of at least nonprogressive disease after 9 weeks after randomization. ${ }^{\mathrm{d} C a l c u l a t e d}$ as the sum of complete responses, partial responses, and stable disease.

The stratified HR for IRF-assessed PFS per RECIST 1.1 was 0.60 (95\% CI, $0.40-0.90$ ) (Fig. 2b). There were 75 progression or death events $(56.4 \%)$ in the atezolizumab plus bevacizumab arm and $38(62.3 \%)$ in the sorafenib arm. The median PFS was 5.7 months (95\% CI, 4.2-8.3) in the atezolizumab plus bevacizumab arm versus 3.2 months (95\% CI, 2.6-4.8) in the sorafenib arm. The proportions of patients without progression or death events at 6 months were 48.5 and $30.5 \%$ in the respective treatment arms. A similar magnitude of PFS benefit with atezolizumab plus bevacizumab was observed when the patients' tumors were assessed using HCC mRECIST criteria. The stratified HR was 0.59 (95\% CI, 0.40-0.89), and the median PFS was 5.7 months (95\% CI, 4.2-8.1) in the atezolizumab plus bevacizumab arm versus 3.2 months $(95 \% \mathrm{CI}$, 2.6-4.8) in the sorafenib arm.

The confirmed ORRs by IRF-assessed RECIST 1.1 were $24.6 \%$ (95\% CI, 17.5-32.9\%) in the atezolizumab plus bevacizumab arm and $6.7 \%$ (95\% CI, 1.9-16.2\%) in the sorafenib arm (Table 2). Disease control rates (total percentage of patients with a confirmed best overall response of complete response, partial response, and stable disease) were 70.0 and $48.3 \%$ in the atezolizumab plus bevacizumab and sorafenib arms, respectively.

When the patients' tumor responses were assessed according to IRF HCC mRECIST criteria, the ORRs were
29.7\% (95\% CI, 21.9-38.4\%) in the atezolizumab plus bevacizumab arm and $8.5 \%$ (95\% CI, 2.8-18.7\%) in the sorafenib arm (Table 2). The disease control rates were similar between the assessment criteria for each treatment arm.

The stratified HR for TTD of QOL was 0.53 (95\% CI, $0.32-0.88$ ) (Fig. 3a); the median TTD was 9.8 months in the atezolizumab plus bevacizumab arm (95\% CI, 6.2 to NR) versus 3.6 months (95\% CI, 1.5-9.8) in the sorafenib arm. The stratified HR for TTD of physical functioning was 0.45 (95\% CI, 0.26-0.78) (Fig. 3b); the median TTD was 13.1 months in the atezolizumab plus bevacizumab arm (95\% CI, 9.7 to NR) versus 5.6 months (95\% CI, 2.1 to NR) in the sorafenib arm. The stratified HR for TTD of role functioning was 0.71 (95\% CI, 0.42-1.23) (Fig. 3c); the median TTD was not reached in either arm.

The median treatment durations were 6.0 months for atezolizumab, 5.5 months for bevacizumab (range for both, $0-16$ months), and 2.8 months (range $0-14$ months) for sorafenib. At least $1 \mathrm{AE}$ was reported by 130 patients (98.5\%) in the atezolizumab plus bevacizumab arm and $56(96.6 \%)$ in the sorafenib arm (Table 3). Treatmentrelated AEs of all grades occurred in 119 patients (90.2\%) receiving atezolizumab plus bevacizumab and 54 (93.1\%) receiving sorafenib. SAEs occurred in 40 patients (30.3\%) in the atezolizumab plus bevacizumab arm and $12 \mathrm{pa}$ - 
Fig. 3. Secondary patient-reported outcomes. Kaplan-Meier analysis of time to deterioration in patient-reported QOL (a), physical functioning (b), and role functioning (c) in the intention-totreat Chinese subpopulation. QOL, quality of life; HR, hazard ratio.

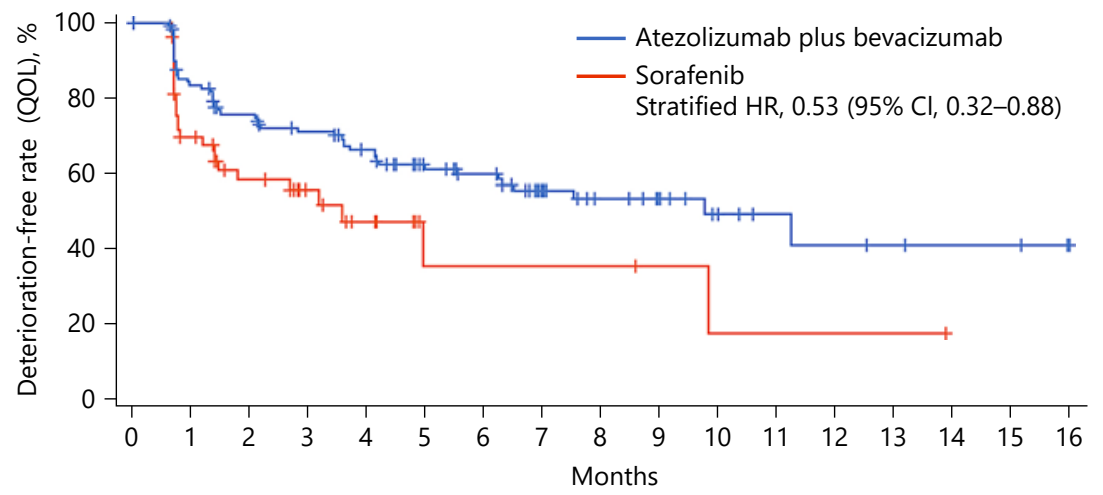

No. at risk

$\begin{array}{llllllllllllllllll}\text { Atezo + Bev } & 133 & 100 & 84 & 76 & 67 & 52 & 44 & 29 & 23 & 17 & 10 & 6 & 5 & 4 & 3 & 3 & \mathrm{NE}\end{array}$ $\begin{array}{llllllllllllllllllll}\text { a } & \text { Sorafenib } & 61 & 35 & 23 & 14 & 9 & 3 & 3 & 3 & 3 & 2 & 1 & 1 & 1 & 1 & \text { NE } & \text { NE } & \text { NE }\end{array}$

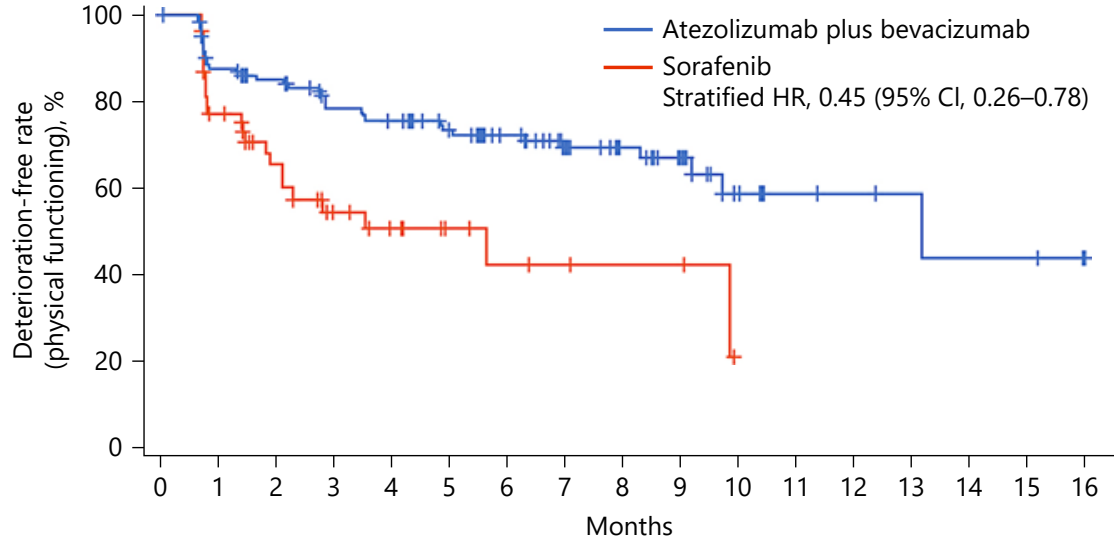

No. at risk

$\begin{array}{llllllllllllllllll}\text { Atezo + Bev } & 133 & 105 & 94 & 81 & 77 & 67 & 55 & 39 & 30 & 22 & 10 & 6 & 5 & 4 & 3 & 3 & \text { NE }\end{array}$

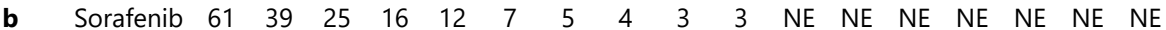

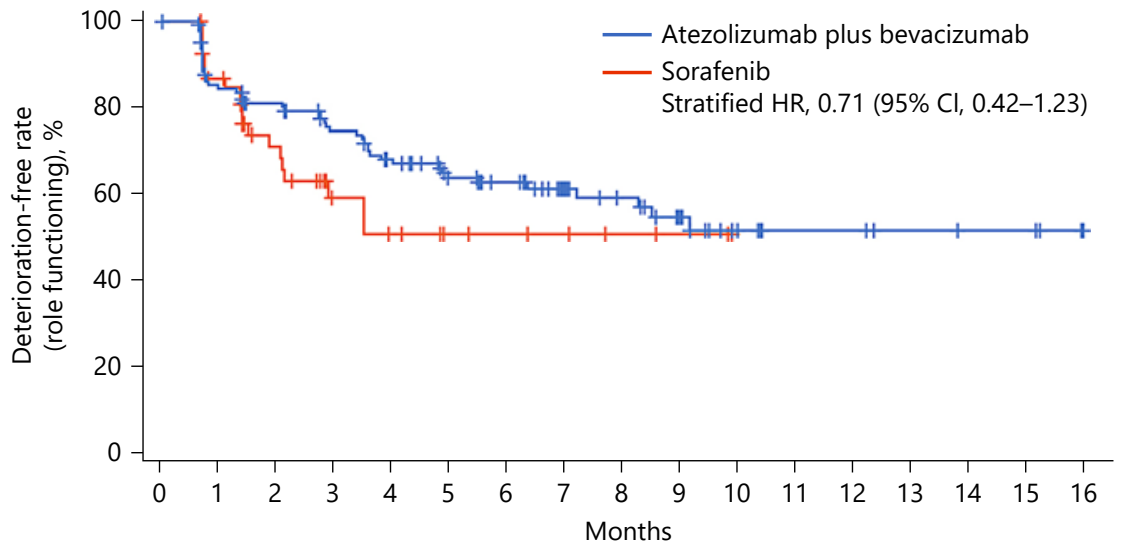

No. at risk

$\begin{array}{llllllllllllllllll}\text { Atezo + Bev } & 133 & 101 & 93 & 81 & 71 & 56 & 46 & 33 & 27 & 20 & 11 & 7 & 7 & 5 & 4 & 4 & \mathrm{NE}\end{array}$

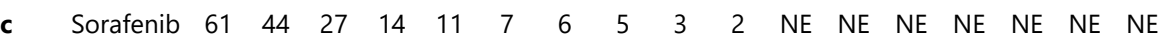


Table 3. Safety summary

\begin{tabular}{|c|c|c|}
\hline & $\begin{array}{l}\text { Atezolizumab } \\
\text { plus bevacizumab } \\
(n=132)\end{array}$ & $\begin{array}{l}\text { Sorafenib } \\
(n=58)\end{array}$ \\
\hline Total number of reported AEs of any cause & 1,251 & 552 \\
\hline \multicolumn{3}{|l|}{ All-cause AEs, $n(\%)$} \\
\hline Any grade & $130(98.5)$ & $56(96.6)$ \\
\hline Grade 3 or $4^{\mathrm{a}}$ & $78(59.1)$ & $27(46.6)$ \\
\hline Grade 5 & $3(2.3)^{b}$ & $2(3.4)^{c}$ \\
\hline SAEs & $40(30.3)$ & $12(20.7)$ \\
\hline AEs leading to withdrawal from any study treatment & $10(7.6)$ & $1(1.7)$ \\
\hline Withdrawal from atezolizumab and bevacizumab & $2(1.5)$ & 0 \\
\hline AEs leading to dose interruption of any study treatment & $62(47.0)$ & $17(29.3)$ \\
\hline AEs leading to dose reduction of sorafenib ${ }^{\mathrm{d}}$ & 0 & $12(20.7)$ \\
\hline \multicolumn{3}{|l|}{ TRAEs, $n(\%)$} \\
\hline Any grade & $119(90.2)$ & $54(93.1)$ \\
\hline Grade 3 or $4^{\mathrm{a}}$ & $58(43.9)$ & $22(37.9)$ \\
\hline Grade 5 & $2(1.5)^{\mathrm{e}}$ & $1(1.7)^{f}$ \\
\hline Treatment-related SAEs & $26(19.7)$ & $6(10.3)$ \\
\hline \multicolumn{3}{|l|}{ AESIs for atezolizumab, $n(\%)$} \\
\hline Any grade & $95(72.0)$ & $51(87.9)$ \\
\hline Grade 3 or $4^{\mathrm{a}}$ & $31(23.5)$ & $14(24.1)$ \\
\hline Grade 5 & 0 & 0 \\
\hline
\end{tabular}

AESI, adverse event of special interest; SAE, serious adverse event; TRAE, treatment-related adverse event.

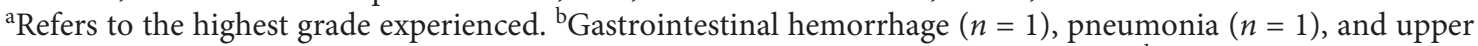
gastrointestinal hemorrhage $(n=1)$. ${ }^{\mathrm{C}}$ Cerebral hemorrhage $(n=1)$ and death $(n=1)$. ${ }^{\mathrm{d}}$ Dose modification of atezolizumab or bevacizumab was not permitted. ${ }^{\mathrm{e}} \mathrm{Gastrointestinal} \mathrm{hemorrhage}(n=1)$ and upper gastrointestinal hemorrhage $(n=1) .{ }^{\mathrm{f} C e r e b r a l ~ h e m o r r h a g e ~}(n=1)$.

tients $(20.7 \%)$ in the sorafenib arm; treatment-related SAEs occurred in $26(19.7 \%)$ and $6(10.3 \%)$ patients in the respective arms.

All-cause AEs of grade 3-4 severity occurred in 78 atezolizumab plus bevacizumab-treated patients (59.1\%) and 27 sorafenib-treated patients $(46.6 \%)$ (Table 3$)$. The most common grade $3 / 4$ event with atezolizumab plus bevacizumab was hypertension in 20 patients (15.2\%; Table 4 ), followed by increased $\gamma$-glutamyl transferase, increased aspartate aminotransferase, and proteinuria (8 patients [6.1\%] each). With sorafenib, the most common grade $3 / 4$ AEs were hypertension in 7 patients $(12.1 \%$; Table 4) and palmar-plantar erythrodysesthesia and increased $\gamma$-glutamyl transferase (4 patients [6.9\%] each). Grade 3/4 treatment-related AEs occurred in 58 patients $(43.9 \%)$ and 22 patients (37.9\%) in the respective treatment arms (Table 3 ). AEs leading to death occurred in 3 patients $(2.3 \%)$ receiving atezolizumab plus bevacizumab (gastrointestinal hemorrhage, upper gastrointestinal hemorrhage [both judged by the investigator to be treatment related], and pneumonia) and in 2 patients (3.4\%) receiving sorafenib (treatment-related cerebral hemorrhage and nonspecified death).

AEs led to withdrawal from any study treatment in 10 patients $(7.6 \%)$ in the atezolizumab plus bevacizumab arm (of which the AE led to discontinuation of both atezolizumab and bevacizumab in 2 patients [1.5\%]) and in 1 patient (1.7\%) in the sorafenib arm (Table 3). All-cause AESIs for atezolizumab and bevacizumab are listed in the online suppl. data (online suppl. Tables 2,3).

After discontinuing study treatment, 26 patients $(19.5 \%)$ in the atezolizumab plus bevacizumab arm and 25 patients $(41.0 \%)$ in the sorafenib arm of the ITT population received follow-up systemic therapy for HCC (online suppl. Table 4 ). The most commonly received poststudy treatment in both study arms was tyrosine kinase inhibitors: 23 patients $(17.3 \%)$ in the atezolizumab plus bevacizumab arm and 13 patients $(21.3 \%)$ in the sorafenib arm. Immunotherapy was received by 1 patient $(0.8 \%)$ in the atezolizumab plus bevacizumab arm and 9 patients $(14.8 \%)$ in the sorafenib arm. 
Table 4. All-cause AEs with incidence of $\geq 10 \%$ in either treatment arm or events of grade $3-5$ with incidence of $\geq 2 \%$ in either arm in the safety population

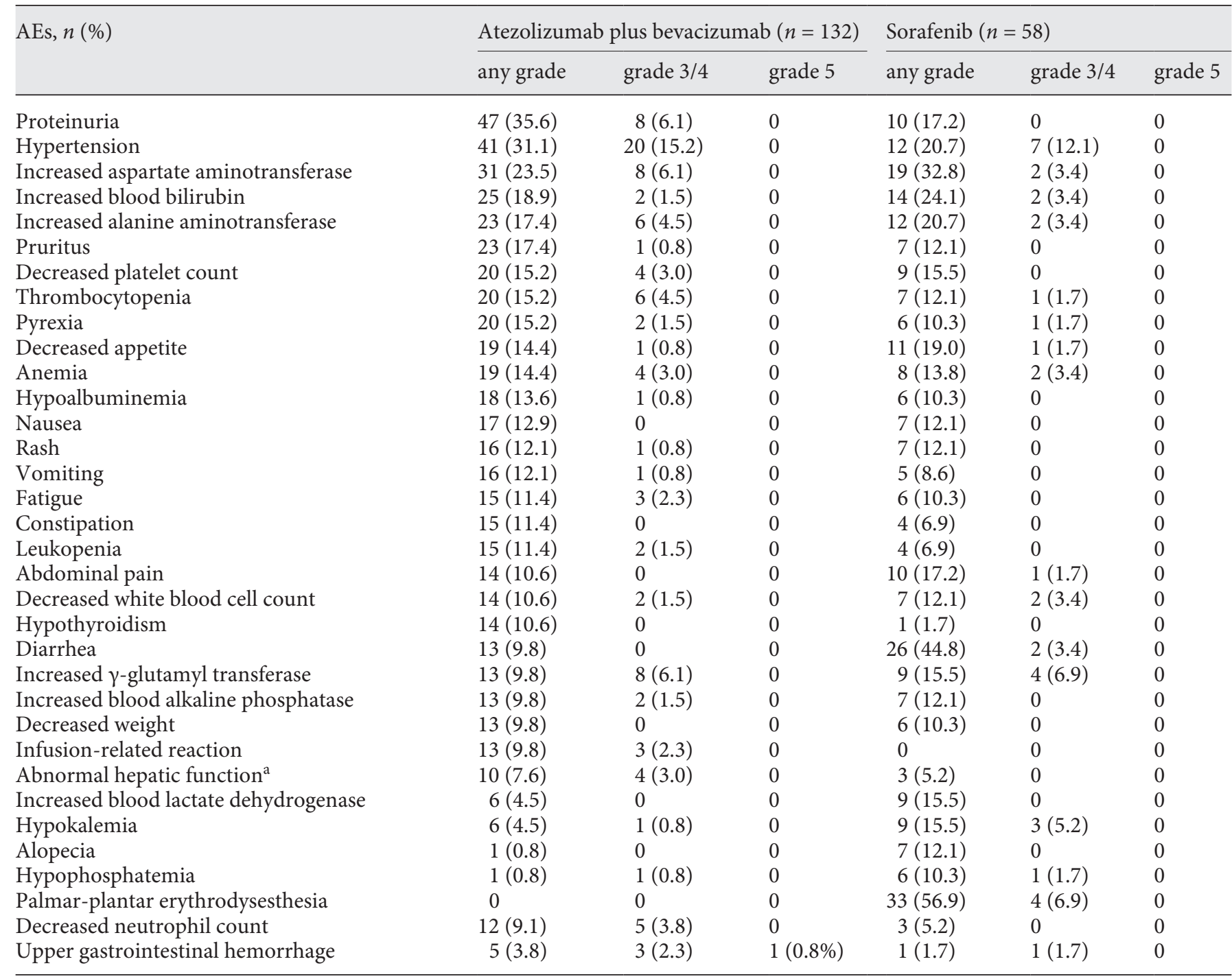

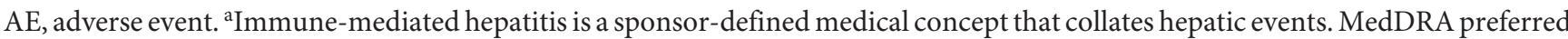
term of "hepatic function abnormal" is categorized under the medical concept of immune-mediated hepatitis.

\section{Discussion}

Atezolizumab plus bevacizumab is the first treatment to demonstrate improved OS over sorafenib [27] since sorafenib was approved to treat unresectable HCC in 2007. Clinically meaningful improvements in OS and PFS were also seen with this combination in the Chinese subpopulation in IMbrave 150. Compared with sorafenib, the HR for OS with atezolizumab plus bevacizumab was 0.44 (95\% CI, 0.25-0.76). The median PFS was 5.7 months in the atezolizumab plus bevacizumab arm versus 3.2 months in the sorafenib arm (HR, 0.60; 95\% CI, 0.40 $0.90)$. The confirmed ORR was 24.6 and $6.7 \%$ in the atezolizumab plus bevacizumab and sorafenib arms, respectively. This treatment combination also resulted in clinically meaningful delays in the deterioration of patient-reported QOL and functioning, compared with sorafenib. These clinical benefits were observed even though more of the Chinese patients had HBV infection ( 85 vs. $48 \%$ of the global population) and poor prognostic factors including Barcelona Clinic Liver Cancer stage C disease ( 89 vs. $82 \%$ ), MVI and/or EHS ( 85 vs. $75 \%$ ), and 
AFP concentrations $\geq 400 \mathrm{ng} / \mathrm{mL}$ ( 46 vs. $37 \%$ ), than the global study population [27]. The efficacy and safety results in the Chinese subpopulation were generally consistent with the findings in the global study population [27].

Overall, the spectrum, incidence, and severity of AEs observed with the combination were consistent with the known safety profile of each agent and the underlying disease. As in the global IMbrave150 population [27], the combination of atezolizumab and bevacizumab was generally well tolerated in Chinese patients and toxicities were manageable. The differences in the incidence of safety events between treatment arms, including the higher incidence of serious and grade $3 / 4$ AEs reported with atezolizumab plus bevacizumab, should be viewed in the context of the different treatment durations in each arm. Proteinuria and hypertension were the most common AEs reported with atezolizumab plus bevacizumab, but most cases were mild in severity, and their incidence rates were comparable to those historically observed with bevacizumab in Chinese patients [32]. As expected, given the underlying liver disease in this study population, most hepatic events in the atezolizumab plus bevacizumab arm were laboratory abnormalities (increased aspartate aminotransferase, alanine aminotransferase, blood bilirubin, and $\gamma$-glutamyl transferase), which were manageable without steroid treatment, drug discontinuation, or dose interruption, as were the AESIs. HBV-associated T regulator cell accumulation compromises viral control while maintaining a tolerogenic environment in the liver [33]. It also contributes to microRNA-34a-C-C motif chemokine ligand 22 signaling-induced intravenous metastasis, resulting in poor prognosis in patients with HCC. However, additional investigation showed that the efficacy and tolerability of atezolizumab plus bevacizumab in Chinese patients with HBV-related HCC in IMbrave150 were consistent with results reported for the global ITT population and the data presented here [34].

Bleeding (including fatal events) is a known $\mathrm{AE}$ with bevacizumab, and before enrollment, patients had to undergo endoscopy to detect varices, which were treated as needed according to local standards of care [27]. Upper gastrointestinal bleeding, a common and life-threatening complication in patients with cirrhosis and HCC, occurred in 5 Chinese patients (4\%) in the atezolizumab plus bevacizumab arm (at grade 5 severity in 1 patient [1\%]) and in 1 patient (2\%) in the sorafenib arm. These rates are consistent with those reported in other trials of bevacizumab for HCC $[35,36]$.

The presence of antidrug antibodies (ADAs) against atezolizumab has been noted for IMbrave150. Due to the small size of the Chinese subpopulation, we are unable to draw any conclusions around the possible relationship between ADAs and efficacy or safety in these patients. Patient safety management is not impacted by ADA status and does not change the overall benefit/risk profile of atezolizumab. Treating physicians should continue to manage toxicities per label guidelines. A potential study limitation is the open-label design of this phase 3 study that was used to spare patients from 2 placebo infusions; consequently, blinded independent review of imaging for the co-primary endpoint of PFS was conducted to minimize the potential bias associated with the open-label design.

In conclusion, clinically meaningful improvements in OS and PFS over sorafenib were observed with atezolizumab plus bevacizumab in the IMbrave150 Chinese subpopulation with unresectable HCC who had not received prior systemic treatment. Atezolizumab plus bevacizumab should therefore be considered to be a practicechanging treatment for Chinese patients with unresectable HCC.

\section{Acknowledgements}

We thank the participating patients and their families and the nurses, research coordinators, data managers, and clinical study site investigators. Medical writing assistance was provided by Samantha Santangelo, PhD, of Health Interactions, Inc., and funded by F. Hoffmann-La Roche, Ltd.

\section{Statement of Ethics}

The study (NCT03434379) was conducted in accordance with Good Clinical Practice guidelines defined by the International Council on Harmonisation, the 1964 Helsinki Declaration, and local laws. The protocol and amendments were approved by the relevant Institutional Review Boards at all centers. All patients provided written informed consent.

\section{Conflict of Interest Statement}

T.Y. has received honoraria from Bristol Myers Squibb and MSD Oncology and served in a consulting or advisory role for Bristol Myers Squibb. L.L. is an employee of Roche. S.H. is an employee of Genentech. D.-Z.X. is an employee of Roche. S.M. is an employee of, and owns stock in, F. Hoffmann-La Roche. Y.W. is an employee of Roche. H.S. is an employee of Roche. A.-L.C. has received honoraria from AstraZeneca, Bayer Yakuhin, Eisai, Genentech/Roche, and Lilly, and has served in a consulting or advisory role for AstraZeneca; Bayer Schering Pharma; BeiGene; Bristol Myers Squibb; CSR Pharma Group; Eisai; Genentech/Roche; IQVIA; MSD; Novartis; and Ono Pharmaceutical. Others claimed no conflicts of interest. A.-L.C. is an associate editor of the journal Liver Cancer. 


\section{Funding Sources}

This study was sponsored by F. Hoffmann-La Roche, Ltd./Genentech, Inc.

\section{Author Contributions}

S.Q., Z.R., L.L., S.H., D.-Z.X., S.M, Y.W., H.S., and A.-L.C. made substantial contributions to the study conception and design, data analysis, and interpretation. All authors contributed to the provision of study material or patients, collection and assembly of data, manuscript writing, and final approval of the manuscript, and are accountable for all aspects of the work.

\section{Data Sharing Statement}

Qualified researchers may request access to individual patient level data through the clinical study data request platform (https:// vivli.org/). Further details on Roche's criteria for eligible studies are available from https://vivli.org/members/ourmembers/. For further details on Roche's Global Policy on the Sharing of Clinical Information and how to request access to related clinical study documents, see https://www.roche.com/research_and_development/who_we_are_how_we_work/clinical_trials/our_commitment_to_data_sharing.htm.

\section{References}

1 Zheng R, Qu C, Zhang S, Zeng H, Sun K, Gu $\mathrm{X}$, et al. Liver cancer incidence and mortality in China: temporal trends and projections to 2030. Chinese J Can Res. 2018;30(6):571-9.

2 Yang JD, Hainaut P, Gores GJ, Amadou A, Plymoth A, Roberts LR. A global view of hepatocellular carcinoma: trends, risk, prevention and management. Nat Rev Gastroenterol Hepatol. 2019 Oct;16(10):589-604.

3 Fan JH, Wang JB, Jiang Y, Xiang W, Liang H, Wei WQ, et al. Attributable causes of liver cancer mortality and incidence in China. Asian Pac J Cancer Prev. 2013;14(12):7251-6.

4 El-Serag HB. Hepatocellular carcinoma. N Engl J Med. 2011 Sep;365(12):1118-27.

5 Rawla P, Sunkara T, Muralidharan P, Raj JP. Update in global trends and aetiology of hepatocellular carcinoma. Contemp Oncol. 2018;22(3):141-50.

6 Villanueva A. Hepatocellular carcinoma. N Engl J Med. 2019;380(15):1450-62.

7 Lau WY, Leung TW, Lai BS, Liew CT, Ho SK, Yu SC, et al. Preoperative systemic chemoimmunotherapy and sequential resection for unresectable hepatocellular carcinoma. Ann Surg. 2001 Feb;233(2):236-41.

8 Tanizaki H, Ryu M, Kinoshita T, Kawano N, Konishi M, Cho A, et al. Comparison of clinical features and survival in patients with hepatitis $B$ and $C$ virus-related hepatocellular carcinoma. Jpn J Clin Oncol. 1997 Apr;27(2): 67-70.

9 Xue X, Liao W, Xing Y. Comparison of clinical features and outcomes between HBV-related and non-B non-C hepatocellular carcinoma. Infect Agent Cancer. 2020;15:11.

10 National Comprehensive Cancer Network. NCCN clinical practice guidelines in oncology. Hepatobiliary Cancers. V1.2020. Available from: https://www.nccn.org/professionals/physician_gls/pdf/hepatobiliary.pdf.

11 Foerster F, Galle PR. Comparison of the current international guidelines on the management of HCC. JHEP Rep. 2019 Aug 1;1(2): $114-9$.
12 Zhou J, Sun HC, Wang Z, Cong WM, Wang $\mathrm{JH}$, Zeng MS, et al. Guidelines for diagnosis and treatment of primary liver cancer in China (2017 edition). Liver Cancer. 2018 Sep; 7(3):235-60.

13 Qin S, Cheng Y, Liang J, Shen L, Bai Y, Li J, et al. Efficacy and safety of the FOLFOX4 regimen versus doxorubicin in Chinese patients with advanced hepatocellular carcinoma: a subgroup analysis of the EACH study. Oncologist. 2014;19(11):1169-78.

14 Kudo M, Finn RS, Qin S, Han KH, Ikeda K, Piscaglia F, et al. Lenvatinib versus sorafenib in first-line treatment of patients with unresectable hepatocellular carcinoma: a randomised phase 3 non-inferiority trial. Lancet. 2018 Mar 24;391(10126):1163-73.

15 El-Khoueiry AB, Sangro B, Yau T, Crocenzi TS, Kudo M, Hsu C, et al. Nivolumab in patients with advanced hepatocellular carcinoma (CheckMate 040): an open-label, noncomparative, phase $1 / 2$ dose escalation and expansion trial. Lancet. 2017;389(10088): 2492-502.

16 Zhu AX, Finn RS, Edeline J, Cattan S, Ogasawara S, Palmer D, et al. Pembrolizumab in patients with advanced hepatocellular carcinoma previously treated with sorafenib (KEYNOTE-224): a non-randomised, openlabel phase 2 trial. Lancet Oncol. 2018 Jun 1; 19(7):940-52.

17 Akinleye A, Rasool Z. Immune checkpoint inhibitors of PD-L1 as cancer therapeutics. J Hematol Oncol. 2019 Sep 5;12(1):92.

18 Flynn MJ, Sayed AA, Sharma R, Siddique A, Pinato DJ. Challenges and opportunities in the clinical development of immune checkpoint inhibitors for hepatocellular carcinoma. Hepatology. 2019 May;69(5):2258-70.

19 Lee MS, Ryoo BY, Hsu CH, Numata K, Stein $\mathrm{S}$, Verret W, et al. Atezolizumab with or without bevacizumab in unresectable hepatocellular carcinoma (GO30140): an open-label, multicentre, phase $1 \mathrm{~b}$ study. Lancet Oncol. 2020;21(6):808-20.
20 Yau T, Park JW, Finn RS, Cheng A-L, Mathurin P, Edeline J, et al. A randomized, multi-center phase III study of nivolumab vs sorafenib as first-line treatment in patients with advanced hepatocellular carcinoma. Ann Oncol. 2019;30(Suppl 5). Abstract LBA38_PR.

21 Finn RS, Ryoo BY, Merle P, Kudo M, Bouattour M, Lim HY, et al. Pembrolizumab as second-line therapy in patients with advanced hepatocellular carcinoma in KEYNOTE-240: a randomized, double-blind, phase III trial. J Clin Oncol. 2020 Jan 20;38(3):193-202.

22 Roland CL, Dineen SP, Lynn KD, Sullivan LA, Dellinger MT, Sadegh L, et al. Inhibition of vascular endothelial growth factor reduces angiogenesis and modulates immune cell infiltration of orthotopic breast cancer xenografts. Mol Cancer Ther. 2009;8(7):1761-71.

23 Motz GT, Santoro SP, Wang LP, Garrabrant T, Lastra RR, Hagemann IS, et al. Tumor endothelium FasL establishes a selective immune barrier promoting tolerance in tumors. Nat Med. 2014;20(6):607-15.

24 Voron T, Colussi O, Marcheteau E, Pernot S, Nizard M, Pointet AL, et al. VEGF-A modulates expression of inhibitory checkpoints on CD8+ T cells in tumors. J Exp Med. 2015; 212(2):139-48.

25 Wallin JJ, Bendell JC, Funke R, Sznol M, Korski K, Jones S, et al. Atezolizumab in combination with bevacizumab enhances antigen-specific T-cell migration in metastatic renal cell carcinoma. Nat Commun. 2016;7:12624.

26 Hegde PS, Wallin JJ, Mancao C. Predictive markers of anti-VEGF and emerging role of angiogenesis inhibitors as immunotherapeutics. Semin Cancer Biol. 2018;52(Pt 2):11724.

27 Finn RS, Qin S, Ikeda M, Galle PR, Ducreux M, Kim TY, et al. Atezolizumab plus bevacizumab in unresectable hepatocellular carcinoma. N Engl J Med. 2020 May 14;382(20): 1894-905. 
28 Llovet JM, Ricci S, Mazzaferro V, Hilgard P, Gane E, Blanc JF, et al. Sorafenib in advanced hepatocellular carcinoma. N Engl J Med. 2008 Jul 24;359(4):378-90.

29 Cheng AL, Kang YK, Chen Z, Tsao CJ, Qin S, Kim JS, et al. Efficacy and safety of sorafenib in patients in the Asia-Pacific region with advanced hepatocellular carcinoma: a phase III randomised, double-blind, placebo-controlled trial. Lancet Oncol. 2009 Jan;10(1): 25-34.

30 Johnson PJ, Qin S, Park JW, Poon RT, Raoul JL, Philip PA, et al. Brivanib versus sorafenib as first-line therapy in patients with unresectable, advanced hepatocellular carcinoma: results from the randomized phase III BRISKFL study. J Clin Oncol. 2013 Oct 1;31(28): 3517-24.
31 Cainap C, Qin S, Huang WT, Chung IJ, Pan $\mathrm{H}$, Cheng $\mathrm{Y}$, et al. Linifanib versus sorafenib in patients with advanced hepatocellular carcinoma: results of a randomized phase III trial. J Clin Oncol. 2015 Jan 10;33(2):172-9.

32 Tsai CM, Au JS, Chang GC, Cheng AC, Zhou C, Wu YL. Safety and efficacy of first-line bevacizumab with chemotherapy in Asian patients with advanced nonsquamous NSCLC: results from the phase IV MO19390 (SAiL) study. J Thorac Oncol. 2011 Jun;6(6):1092-7.

33 Yang P, Markowitz GJ, Wang XF. The hepatitis $\mathrm{B}$ virus-associated tumor microenvironment in hepatocellular carcinoma. Natl Sci Rev. 2014 Jul;1(3):396-412.

34 Qin S, Ren Z, Zhao H, Wang B, Bai Y, Gu S, et al. Abstract 8208: efficacy, patient-reported outcomes (PRO) and safety of atezolizumab + bevacizumab vs sorafenib in patients with hepatitis B virus (HBV)-related unresectable hepatocellular carcinoma (HCC) in IMbrave 150. Chinese Society of Clinical Oncology 23rd Annual Meeting. 2020 Sep.

35 Finn RS, Bentley G, Britten CD, Amado R, Busuttil RW. Targeting vascular endothelial growth factor with the monoclonal antibody bevacizumab inhibits human hepatocellular carcinoma cells growing in an orthotopic mouse model. Liver Int. 2009 Feb;29(2):28490.

36 Boige V, Malka D, Bourredjem A, Dromain C, Baey C, Jacques N, et al. Efficacy, safety, and biomarkers of single-agent bevacizumab therapy in patients with advanced hepatocellular carcinoma. Oncologist. 2012;17(8): 1063-72. 